\title{
ARTICLE ClC-3 promotes angiotensin II-induced reactive oxygen species production in endothelial cells by facilitating Nox2 NADPH oxidase complex formation
}

\author{
Guo-zheng Liang ${ }^{1}$, Li-min Cheng ${ }^{1}$, Xing-feng Chen ${ }^{1}$, Yue-jiao Li $^{1}$, Xiao-long $\mathrm{Li}^{1}$, Yong-yuan Guan ${ }^{1}$ and Yan-hua Du $^{1}$
}

\begin{abstract}
Recent evidence suggests that $\mathrm{ClC}-3$, a member of the $\mathrm{CIC}$ family of $\mathrm{Cl}^{-}$channels or $\mathrm{Cl}^{-} / \mathrm{H}^{+}$antiporters, plays a critical role in NADPH oxidase-derived reactive oxygen species (ROS) generation. However, the underling mechanisms remain unclear. In this study we investigated the effects and mechanisms of ClC-3 on NADPH oxidase activation and ROS generation in endothelial cells. Treatment with angiotensin II (Ang II, $1 \mu \mathrm{mol} / \mathrm{L}$ ) significantly elevated ClC-3 expression in cultured human umbilical vein endothelial cells (HUVECs). Furthermore, Ang II treatment increased ROS production and NADPH oxidase activity, an effect that could be significantly inhibited by knockdown of $\mathrm{CIC}-3$, and further enhanced by overexpression of CIC-3. SA- $\beta$-galactosidase staining showed that CIC-3 silencing abolished Ang II-induced HUVEC senescence, whereas CIC-3 overexpression caused the opposite effects. We further showed that Ang II treatment increased the translocation of p47phox and p67phox from the cytosol to membrane, accompanied by elevated Nox2 and p22phox expression, which was significantly attenuated by knockdown of ClC-3 and potentiated by overexpression of CIC-3. Moreover, overexpression of CIC-3 increased Ang II-induced phosphorylation of p47phox and p38 MAPK in HUVECs. Pretreatment with a p38 inhibitor SB203580 abolished CIC-3 overexpression-induced increase in p47phox phosphorylation, as well as NADPH oxidase activity and ROS generation. Our results demonstrate that CIC-3 acts as a positive regulator of Ang II-induced NADPH oxidase activation and ROS production in endothelial cells, possibly via promoting both Nox2/p22phox expression and p38 MAPK-dependent p47phox/p67phox membrane translocation, then increasing Nox2 NADPH oxidase complex formation.
\end{abstract}

Keywords: CIC-3; angiotensin II; oxidative stress; NADPH oxidase; endothelial cell

Acta Pharmacologica Sinica (2018) 39:1725-1734; https://doi.org/10.1038/s41401-018-0072-0

\section{INTRODUCTION}

Reactive oxygen species (ROS) have been implicated in cellular signaling processes and as causes of oxidative stress. Over the past two decades, the term oxidative stress has often been used to describe the effects of ROS derived from NADPH oxidases (Nox), a membrane-localized protein. To date, seven members (Nox1, Nox2, Nox3, Nox4, Nox5, Duox1, and Duox2) of the NADPH oxidase family have been identified and shown to be involved in a surprisingly varied array of functions [1, 2]. However, despite tremendous advances in our knowledge of the role of ROS in cell function, the process of NADPH oxidase activation remains not fully understood.

ClC-3, a member of the $\mathrm{ClC}$ family of $\mathrm{Cl}^{-}$channels or $\mathrm{Cl}^{-} / \mathrm{H}^{+}$ antiporters, is localized to the plasma membrane or intracellular vesicles and is ubiquitously expressed in almost all mammalian cells $[3,4]$. Historically, CIC-3 has been demonstrated to play an important role in the regulation of a variety of physiological activities, including intracellular $\mathrm{pH}$, cell volume, proliferation, differentiation, migration, apoptosis, cerebrovascular remodeling, neointima formation, and atherosclerosis [5-9]. Recently, a correlative link between $\mathrm{CIC}-3$ and NADPH oxidase was made. It was first reported that the activity of NADPH oxidase and Nox2mediated neutrophil functions are impaired in $\mathrm{ClC}-3$ knockout mice [10]. This unexpected involvement of $\mathrm{ClC}-3$ in Nox activation was further demonstrated by the same group with another Nox family member, demonstrating that Nox1 activity and signaling require $\mathrm{ClC}-3$ [11]. Deficiency in ClC-3 inhibits Nox1-derived ROS production induced by cytokines in the endosomes of vascular smooth muscle cells [11]. The dependence of NADPH oxidase activity on $\mathrm{ClC}-3$ was also recently described in human blood eosinophils [12]. Consistent with these findings, our recent study showed that a lack of $\mathrm{ClC}-3$ inhibited angiotensin II (Ang II)induced endothelial progenitor cell apoptosis by suppressing ROS generation from NADPH oxidase [13]. Although these findings reveal the fact that $\mathrm{ClC}-3$ controls Nox in various cell types, the exact underlying mechanisms through which $\mathrm{ClC}-3$ regulates $\mathrm{NADPH}$ oxidase/ROS generation remain unclear.

It is well known that endothelial oxidative stress plays an important role in vascular dysfunction and damage, in which NADPH oxidase is a major source of excessive ROS production [14]. Considering that the involvement of $\mathrm{ClC}-3$ in the regulation of endothelial NADPH oxidase is not known, the aim of our study

\footnotetext{
${ }^{1}$ Department of Pharmacology, Cardiac \& Cerebral Vascular Research Center, Zhongshan School of Medicine, Sun Yat-Sen University, Guangzhou 510080, China Correspondence: Yan-hua Du (duyanhua@mail.sysu.edu.cn)

These authors contributed equally: Guo-zheng Liang, Li-min Cheng.
}

Received: 22 March 2018 Accepted: 10 June 2018

Published online: 5 July 2018 
was to investigate the effect and mechanism of CIC-3 on NADPHderived ROS generation in endothelial cells. Our results demonstrated that Ang Il-induced NADPH oxidase activation in endothelial cells requires the presence of $\mathrm{ClC}-3$. Facilitating the formation of an active Nox2 NADPH complex underlies, at least in part, the upregulating effects of $\mathrm{CIC}-3$ on enzyme activity.

\section{MATERIALS AND METHODS}

Cell isolation and culture

Human umbilical vein endothelial cells (HUVECs) were isolated and cultured as described previously [15]. The experiments were approved by the medical research ethics committee of Sun YatSen University and conducted according to the principles expressed in the Declaration of Helsinki. Informed consents were obtained from all subjects. In brief, HUVECs were harvested from the umbilical vein and digested by $0.125 \%$ trypsin (Sigma-Aldrich, USA) with $0.01 \%$ ethylene glycol-bis ( $\beta$-aminoethyl ether)- $\mathrm{N}_{1} \mathrm{~N}, \mathrm{~N}^{\prime}, \mathrm{N}^{\prime}-$ tetraacetic acid (EGTA). The cells were then cultured in M199 culture medium containing $20 \%$ fetal calf serum, $100 \mathrm{U} / \mathrm{mL}$ penicillin, $100 \mathrm{U} / \mathrm{mL}$ streptomycin, $25 \mathrm{U} / \mathrm{mL}$ heparin, $2 \mathrm{mmol} / \mathrm{L}$

a
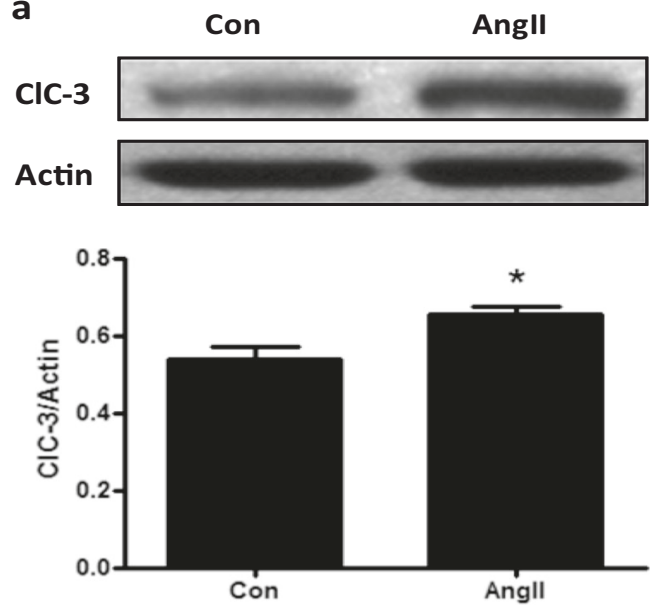

b
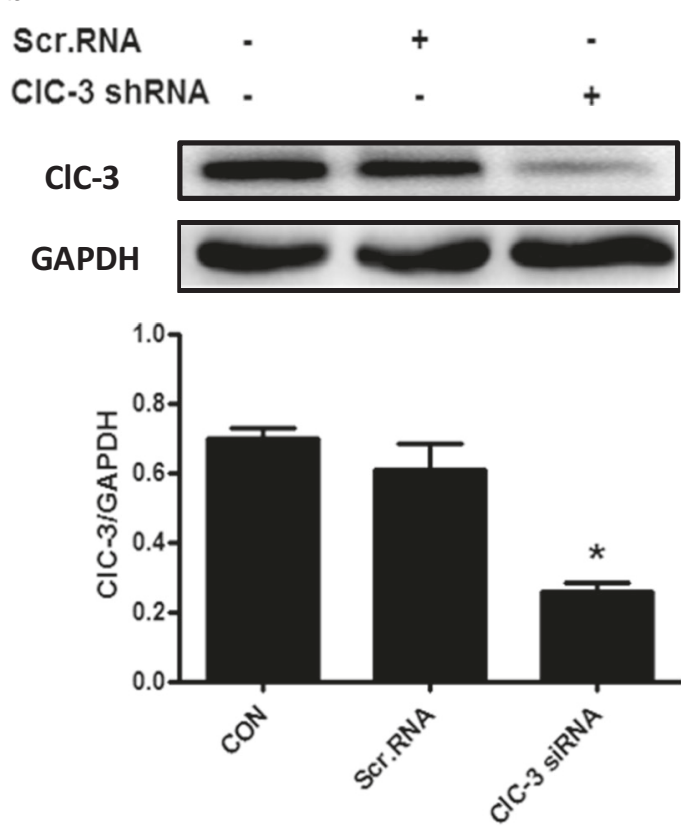

L-glutamine, and $5 \mathrm{ng} / \mathrm{mL}$ p-ECGF at $37^{\circ} \mathrm{C}, 5 \% \mathrm{CO}_{2}$ humidified atmosphere. Cells between passages 4 and 8 were used in this study.

\section{Adenovirus transfection}

Adenovirus (Ad)-ClC-3 shRNA and Ad-ClC-3 cDNA were designed and produced by Sunbio Medical Biotechnology (Shanghai, China). The sequence of the CIC-3 shRNA is 5'-CCACGACTGGTTTATCTIT-3' (NM_001243374.1). The full-length gpCIC-3 cDNA was kindly provided by Dr J.R. Hume, University of Nevada School of Medicine, Reno, NV, USA. Cultured HUVECs at 50\% confluence were infected with Ad encoding CIC-3 shRNA or CIC-3 cDNA for $6 \mathrm{~h}$ and then washed and incubated in fresh medium for an additional $48 \mathrm{~h}$ before the experiment.

Detection of ROS production

ROS levels in Ang II-treated HUVECs were measured using a dihydroethidium (DHE) probe. HUVECs were rinsed with Hank's buffer and then incubated with DHE $(5 \mu \mathrm{mol} / \mathrm{L})$ at $37^{\circ} \mathrm{C}$ for $30 \mathrm{~min}$ in the dark. The fluorescent signal was detected by a fluorescence microscope (Olympus, Tokyo, Japan) at 535-nm excitation and
C
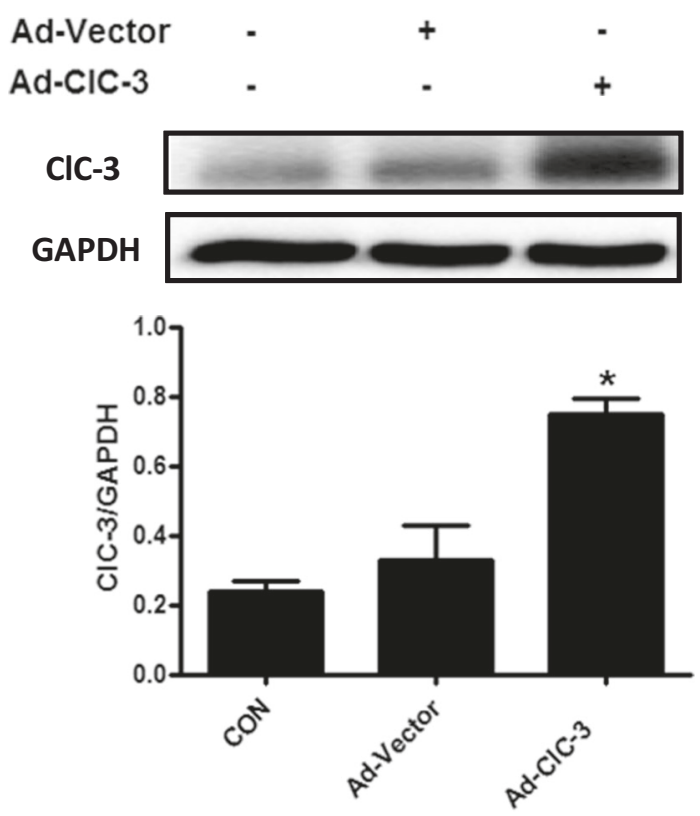
d
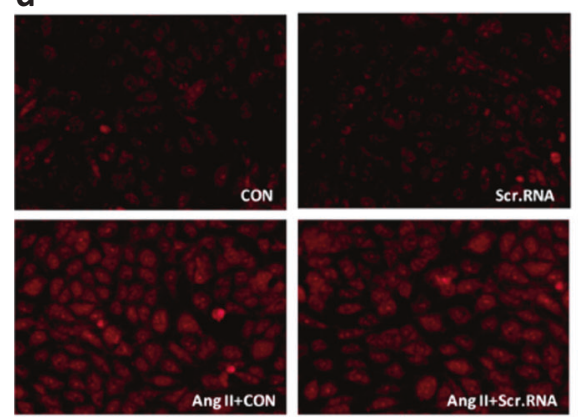

e
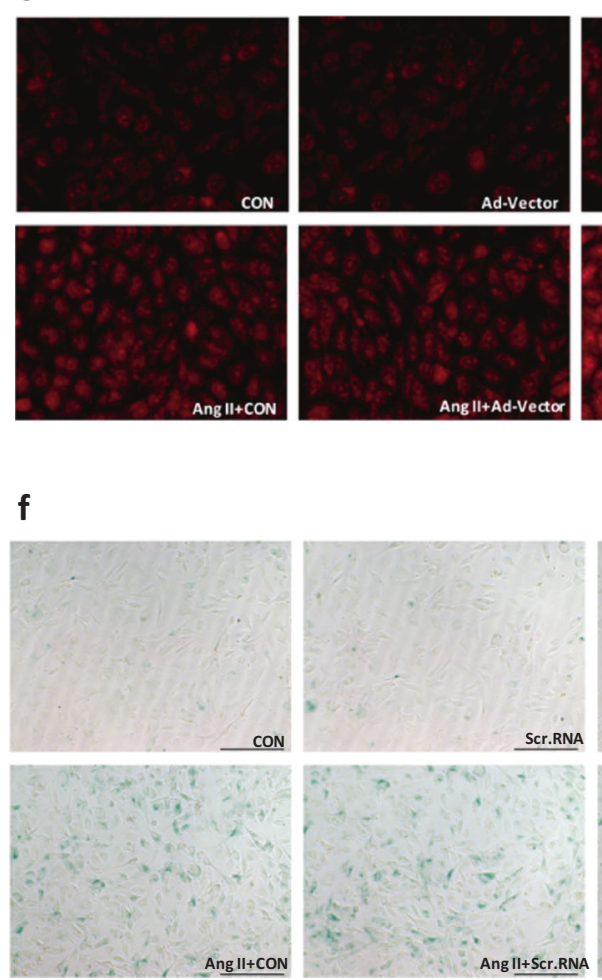

f
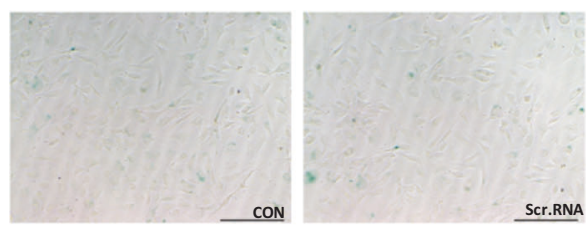
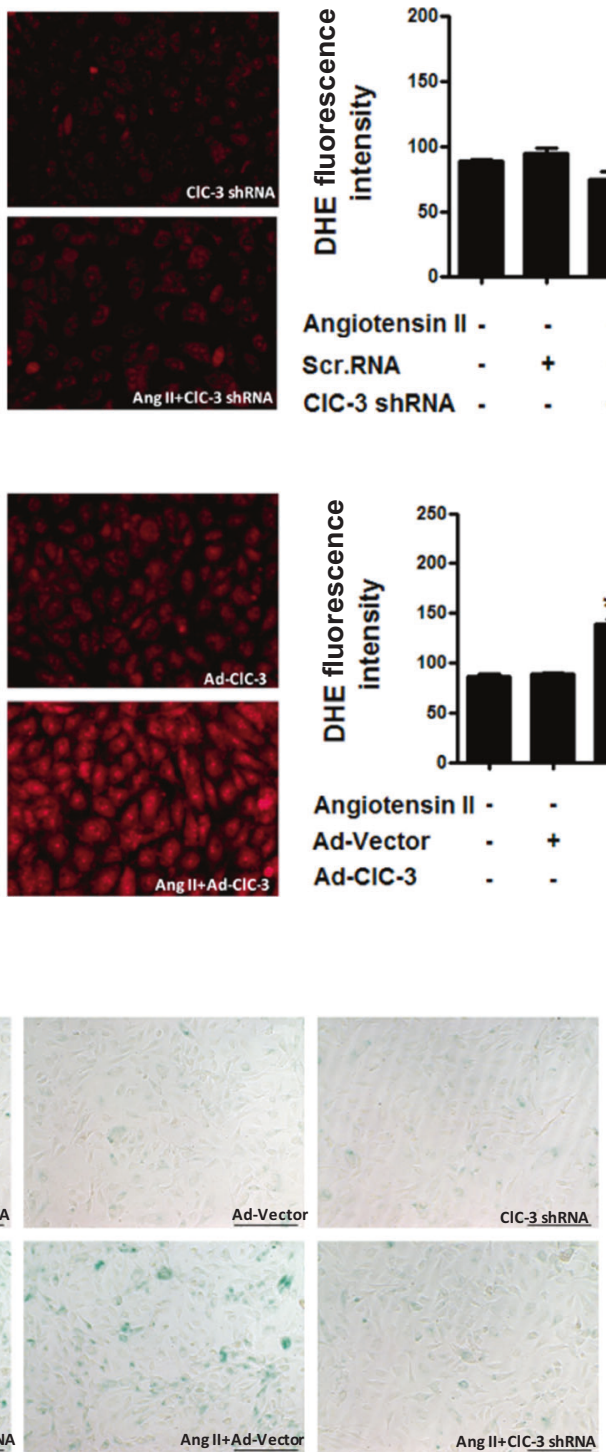
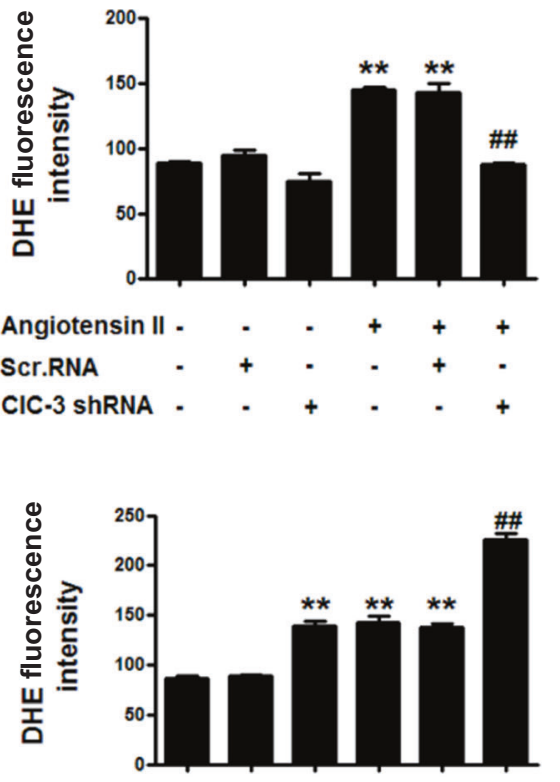

Angiotensin II - $\quad-\quad+\quad+\quad+\quad+$

Ad-Vector - + - - + +

Ad-ClC-3 - $-+\quad-\quad+$

Fig. 1 Effects of CIC-3 on Ang Il-induced ROS production in HUVECs. a Ang II treatment increased CIC-3 expression in HUVECs $\left({ }^{*} P<0.05\right.$ vs. CON. $n=5$ ). $\mathbf{b}$ and $\mathbf{c}$ Effects of adenovirus (Ad)-ClC-3 shRNA and Ad-CIC-3 transfection on the expression of CIC-3 in HUVECs. Cells were transfected with Ad-ClC-3 shRNA (100 MOI) (b) or Ad-CIC-3 (100 MOI) (c) for $48 \mathrm{~h}\left({ }^{*} P<0.05\right.$ vs. CON. $\left.n=4\right)$. $d$ and e Representative images $(\times$ 400) with quantitative analysis of the dihydroethidium (DHE) fluorescence intensity (in arbitrary units) were shown in HUVECs transfected with Ad-CIC-3 shRNA (d) or Ad-CIC-3 (e) upon stimulation with Ang II $(1 \mu \mathrm{mol} / \mathrm{L})$ for $24 \mathrm{~h}$. Ang II-induced ROS production was attenuated by knockdown of CIC-3, and further enhanced by CIC-3 overexpression $\left(n=6,{ }^{* *} P<0.01\right.$ vs. CON, $\# \# P<0.01$ vs. Ang II). f CIC-3 potentiated Ang IIinduced senescence of HUVECs. HUVECs were transfected with Ad-CIC-3 shRNA or Ad-CIC-3 for $48 \mathrm{~h}$ and then were incubated with or without Ang II $(1 \mu \mathrm{mol} / \mathrm{L})$ for $24 \mathrm{~h}$. Representative photomicrographs $(\times 400)$ show the senescence-associated $\beta$-galactosidase $(S A-\beta-G a l)$-positive cells with different treatments $(n=6)$

610-nm emission. The results were quantified using Image-Pro Plus software.

\section{NADPH oxidase activity assay}

The NADPH oxidase activity was measured by lucigenin-enhanced chemiluminescence as described previously [13]. Briefly, for cultured HUVECs, a cell suspension was created by detachment with phosphate buffer $(50 \mathrm{mmol} / \mathrm{L})$ containing EGTA $(1 \mathrm{mmol} / \mathrm{L})$ and protease inhibitor cocktail $(\mathrm{pH} 7.0)$ and sonicated for $10 \mathrm{~s}$ on ice. After incubation with lucigenin $(5 \mu \mathrm{mol} / \mathrm{L}$, Sigma) for $10 \mathrm{~min}$ at $37^{\circ} \mathrm{C}$, the basal relative light units (RLU) of chemiluminescence were read by a luminometer (GLOMAX-20/20, Promega) every $10 \mathrm{~s}$ for $1 \mathrm{~min}$. As soon as NADPH ( $100 \mu \mathrm{mol} / \mathrm{L}$, Sigma) was added to the suspension to start the reaction, the chemiluminescence was read for another $1 \mathrm{~min}$ as the experimental RLU. Then, the basal RLU was subtracted from the total count. The NADPH oxidase activity was calculated from the ratio of mean light units to the total protein level and expressed as arbitrary units.

\section{Senescence-associated $\beta$-galactosidase staining}

Cell senescence was determined by in situ staining for senescence-associated-galactosidase using a senescence cell histochemical staining kit (Cell Signaling Technology, USA). In brief, HUVECs were first fixed for $10 \mathrm{~min}$ at room temperature in fixation buffer. After washing with phosphate-buffered saline (PBS), cells were incubated with a $\beta$-Galactosidase staining solution for $12 \mathrm{~h}$ at $37^{\circ} \mathrm{C}$ in a dry incubator without $\mathrm{CO}_{2}$. The reaction was stopped by the addition of PBS. 


\section{Coimmunoprecipitation}

Cell lysates were incubated with precoupled antibodies bound to protein $\mathrm{G}$ beads overnight at $4{ }^{\circ} \mathrm{C}$. The beads were centrifuged and washed and then boiled in protein sodium dodecyl sulfate sample buffer. Samples were resolved on $8 \%$ sulfate polyacrylamide gel electrophoresis (SDS-PAGE) gels and transferred to polyvinylidene fluoride (PVDF) membranes. The bound proteins were determined by immunoblotting with the indicated antibodies.

Subcellular fraction isolation

Membrane and cytosolic proteins were isolated by using a Qproteome Cell Compartment Kit (QIAGEN, CA, USA) according to the manufacturer's instructions. In brief, the cell suspension was rinsed by PBS and then lysed by ice-cold Extraction Buffer CE1 for the cytoplasm and ice-cold Extraction Buffer CE2 for the membrane.

\section{Western blot}

Western blot was performed according to the protocol as described previously [15]. HUVECs were rinsed with ice-cold PBS and lysed with radioimmunoprecipitation assay buffer lysis buffer containing protease inhibitor cocktail (Merck, Germany). The protein concentration was determined with a BCA kit. A total of $80 \mu \mathrm{g}$ protein was separated with $10 \%$ SDS-PAGE and transformed to PVDF membranes (Millipore, USA). After blocking in 5\% skimmed milk for $1 \mathrm{~h}$ at room temperature, the membranes were incubated with primary antibodies against CIC-3 (Alomone, ISR), Nox2 (gp91phox, Santa Cruz, USA), p22phox (Santa Cruz, USA), p47phox (Santa Cruz, USA), p67phox (Santa Cruz, USA), Rac1 (Santa Cruz, USA), phospho-p47phox (Abcam, USA), p38 MAPK or phospho-p38 MAPK (Cell Signaling Technology, USA) at $4{ }^{\circ} \mathrm{C}$ overnight. Incubation with monoclonal mouse GAPDH (Santa Cruz, USA) or $\mathrm{Na}^{+}-\mathrm{K}^{+}$-ATPase (Santa Cruz, USA) antibody was performed as the loading sample control for the whole cell lysate or plasma membrane fraction, respectively. After incubation with the secondary antibody conjugated to horseradish peroxidase (Cell Signaling Technology, USA) for $1 \mathrm{~h}$ at room temperature, bands were detected with Pierce ECL Western blotting substrate (Thermo Scientific, USA) and quantified with a computer-aided one-dimensional gel analysis system.

\section{Statistical analysis}

All statistical analyses were performed using GraphPad Prism 5. All data were expressed as the mean \pm SEM. A two-tailed Student's $t$ test for independent samples was used to detect significant differences between two groups. One-way analysis of variance followed by Bonferroni multiple comparison test was used to compare differences when there were more than two treatment groups. Values of $P<0.05$ were considered statistically significant.

\section{RESULTS}

CIC-3 potentiates Ang II-induced ROS production

We first determined whether $\mathrm{ClC}-3$ could affect Ang II-induced ROS generation in HUVECs. Ad-ClC-3 shRNA and Ad-ClC-3 were used to silence or overexpress $\mathrm{ClC}-3$, respectively (Fig. 1b, c present the infection efficiency). As shown in Fig. 1, Ang II treatment significantly increased $\mathrm{CIC}-3$ expression in HUVECs (Fig. 1a). ROS production detected by DHE staining was also remarkably increased in cultured HUVECs upon Ang II administration. However, this increase in the ROS level was abolished by the silencing of $\mathrm{ClC}-3$ (Fig. 1d) and was further increased by $\mathrm{ClC}-3$ overexpression (Fig. 1e). ClC-3 overexpression alone by $\mathrm{Ad}$ transfection was sufficient to increase intracellular ROS production even without Ang II stimulation. These results indicate that $\mathrm{CIC}-3$ has an essential role in regulating ROS generation in endothelial cells.

In addition, to further explore whether the contribution of $\mathrm{ClC}-3$ to ROS generation would have an effect on endothelial cell function, we examined the involvement of $\mathrm{ClC}-3$ in the Ang IIinduced senescence of HUVECs, a process in which oxidative stress has been shown to play an important role. $\beta$-galactosidase staining revealed that knockdown of $\mathrm{ClC}-3$ inhibited and $\mathrm{ClC}-3$ overexpression enhanced the HUVEC senescence induced by Ang II (Fig. 1f).

CIC-3 increases Ang II-induced NADPH oxidase activation There is strong evidence that Ang II stimulates ROS formation by activating membrane-bound NADPH oxidase in the vasculature $[13,14]$. Therefore, the roles of $\mathrm{ClC}-3$ in Ang IIinduced NADPH oxidase activation were examined. The results revealed that Ang II augmented the NADPH oxidase activity from $52.10 \pm 1.81$ to $78.99 \pm 4.05 \mathrm{RLU} / \mathrm{s} / \mathrm{mg}$ protein in HUVECs, which was significantly inhibited by the knockdown of $\mathrm{ClC}-3$ to $59.45 \pm 2.600 \mathrm{RLU} / \mathrm{s} / \mathrm{mg}$ protein (Fig. 2a) and further enhanced to $132.9 \pm 10.63 \mathrm{RLU} / \mathrm{s} / \mathrm{mg}$ protein by the overexpression of $\mathrm{ClC}-3$ (Fig. 2b). Moreover, consistent with the ROS production shown in Fig. 1e, the overexpression of $\mathrm{ClC}-3$ alone caused an increase in NADPH oxidase activity without stimulation with Ang II (Fig. 2b).

CIC-3 increases the expression of Nox2 and p22phox In endothelial cells, Nox2 NADPH oxidase has been shown to be the primary source of Ang II-induced $\mathrm{O}_{2}{ }^{-}[16]$. Nox2 is a
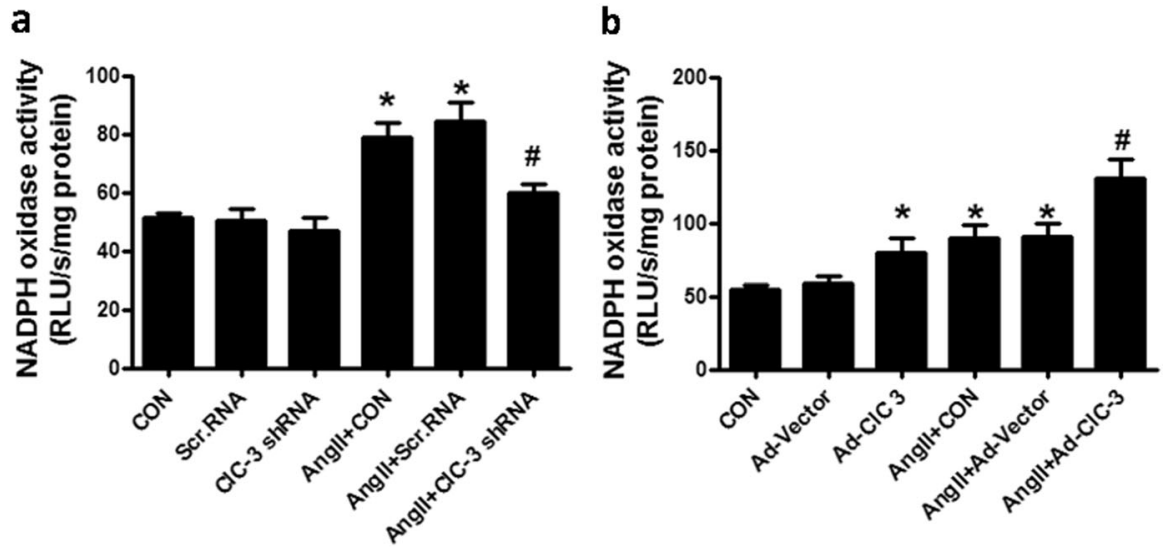

Fig. 2 Effect of CIC-3 on Ang II-induced NADPH oxidase activity in HUVECs. Cells were treated with Ang II ( $1 \mu \mathrm{mol} / \mathrm{L})$ for $24 \mathrm{~h}$. Silencing of CIC-3 inhibited (a), whereas overexpression of CIC-3 promoted (b) Ang Il-induced NADPH oxidase activation $\left(n=6,{ }^{*} P<0.05\right.$ vs. CON, ${ }^{\sharp} P<0.05$ vs. Ang II). RLU indicates the relative light units 


\begin{tabular}{l|llllll} 
Angiotensin II & - & - & - & + & + & + \\
Scr.RNA & - & + & - & - & + & - \\
CIC-3 shRNA & - & - & + & - & - & + \\
NOX2 & \\
p22phox & & & & & & \\
GAPDH
\end{tabular}

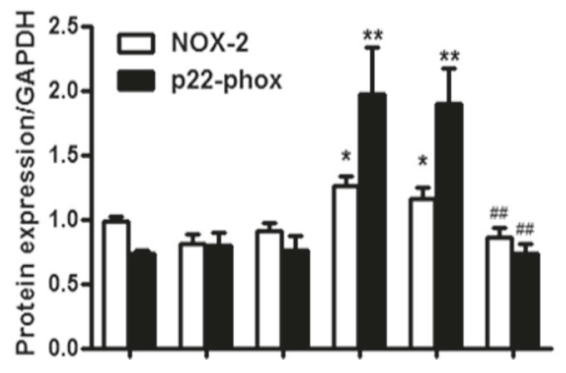

C
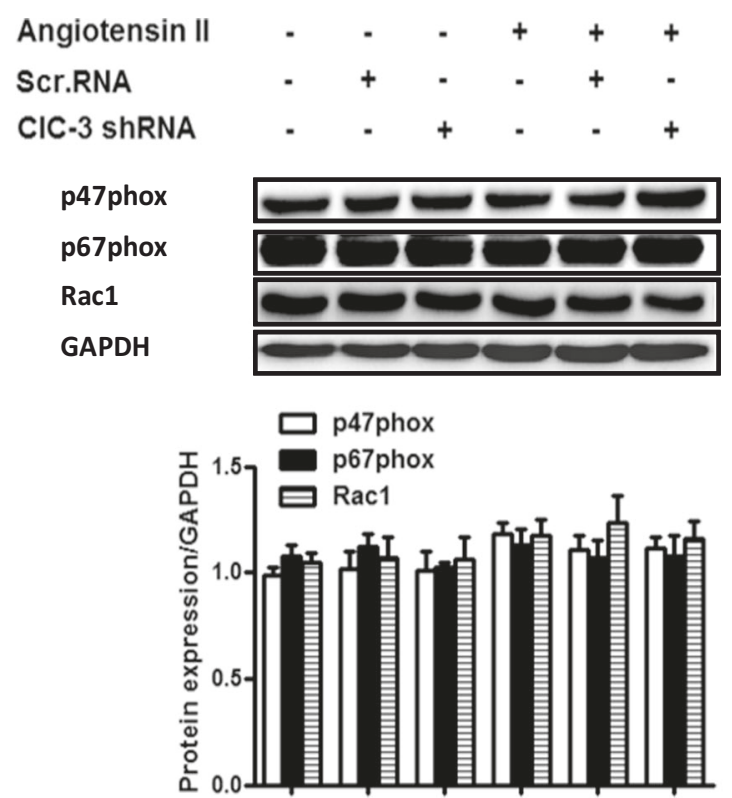

b
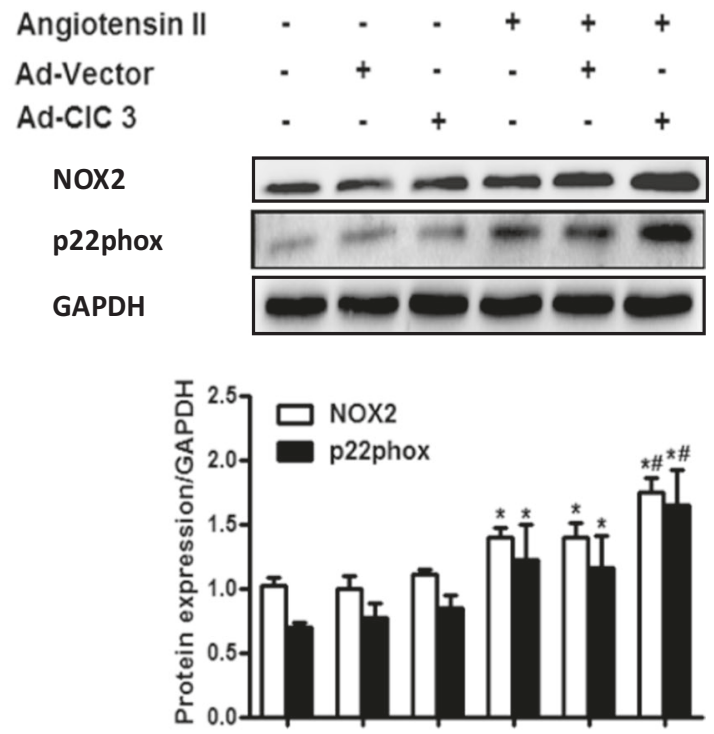

d

$\begin{array}{lllllll}\text { Angiotensin II } & - & - & - & + & + & + \\ \text { Ad-Vector } & - & + & - & - & + & - \\ \text { Ad-CIC-3 } & - & - & + & - & - & +\end{array}$

p47phox

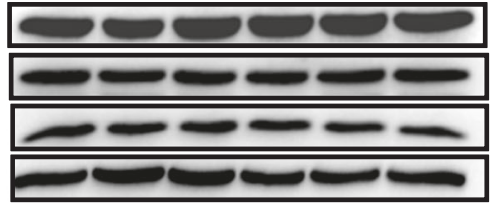

GAPDH

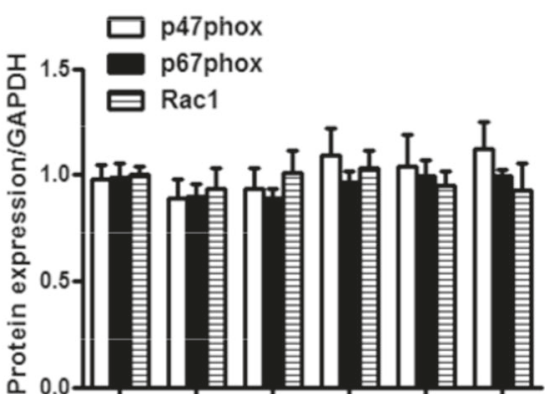

Fig. 3 Effect of $\mathrm{CIC}-3$ on expression levels of NADPH oxidase subunits. $\mathbf{a}$ and $\mathbf{b}$ Ang II $(1 \mu \mathrm{mol} / \mathrm{L})$ treatment for $24 \mathrm{~h}$ increased the expression of Nox2 and p22phox, which was attenuated by knockdown of CIC-3 (a) and enhanced by overexpression of CIC $-3(\mathbf{b})\left(n=6,{ }^{*} P<0.05\right.$ vs. CON, ${ }^{\#} P<0.05$ vs. Ang II, ${ }^{* *} P<0.01$ vs. CON ${ }^{\# \#} P<0.01$ vs. Ang II). $\mathbf{c}$ and $\mathbf{d}$ Knockdown or overexpression of ClC-3 did not alter the expression of p47phox, p67phox and Rac1. $n=6$

multisubunit complex, which consists of the membrane-bound catalytic gp91phox (Nox2) subunit and p22phox subunit, as well as three cytosolic regulatory subunits, p47phox, p67phox, and Rac1 [14]. To understand the mechanism through which $\mathrm{CIC}-3$ regulates NADPH oxidase activity, the expression of these subunits was examined. The results showed that Ang II significantly increased the expression of the membrane-bound subunits Nox2 and p22phox, which was attenuated by the knockdown of $\mathrm{ClC}-3$ and potentiated by the overexpression of $\mathrm{ClC}-3$ in HUVECs (Fig. 3a, b). However, the expression of cytosolic subunits p47phox, p67phox, and Rac1 was not altered either by Ang II or by gene modulation of $\mathrm{ClC}-3$ (Fig. 3c, d).
CIC-3 increases the translocation of p47phox and p67phox

The translocation of p47phox and p67phox from the cytosol to the cell membrane is the key step in the assembly of the NADPH complex and is a crucial mechanism for enzyme activation [17]. We therefore further examined whether $\mathrm{CIC}-3$ regulates $\mathrm{p} 47 \mathrm{phox}$ and p67phox translocation. As shown in Fig. 4a, immunoprecipitation showed that Ang II remarkably increased the p47phox interaction with p22phox and p67phox in HUVECs, which was significantly inhibited by $\mathrm{ClC}-3$ shRNA silencing (Fig. 4a) and further potentiated by $\mathrm{ClC}-3$ overexpression (Fig. 4b). This finding indicates that $\mathrm{ClC}-3$ might facilitate the translocation of p47phox and p67phox to the cell membrane, increasing the interaction 
a

IP:

p22phox
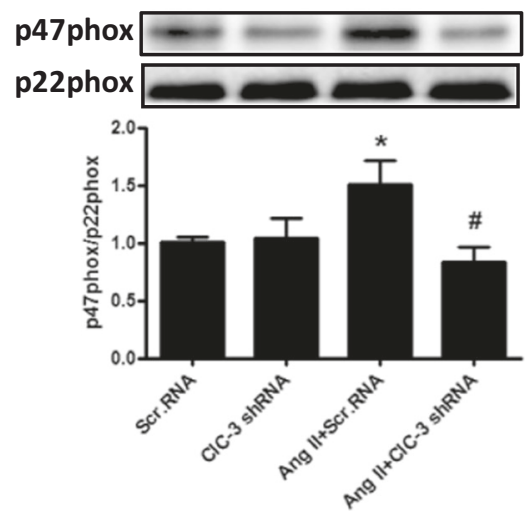

b
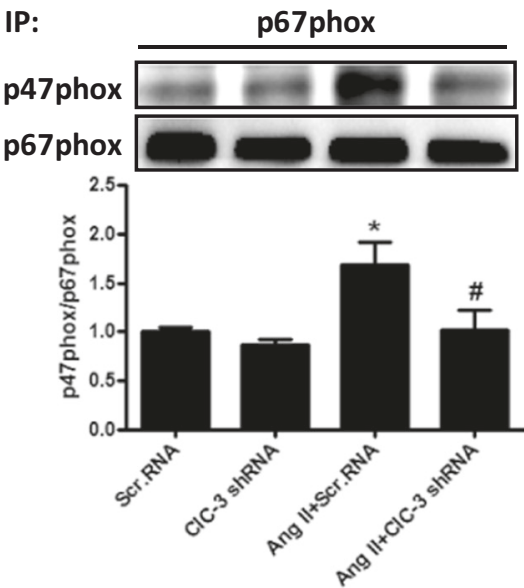

IP:

p22phox

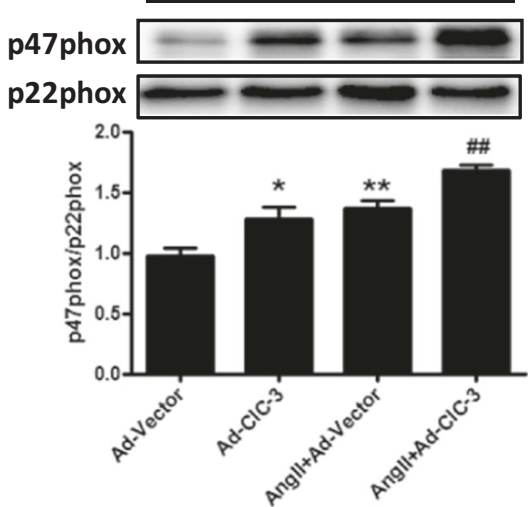

IP: p67phox

p47phox p67phox

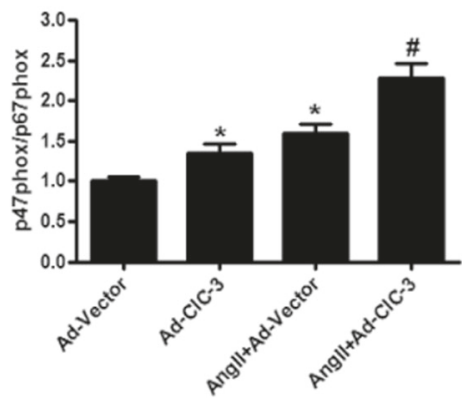

C

d

\begin{tabular}{|c|c|c|c|c|c|c|c|c|c|c|c|c|c|}
\hline Angiotensin II & - & - & - & + & + & + & Angiotensin II & $\cdot$ & $\cdot$ & $\cdot$ & + & + & + \\
\hline Scr.RNA & $\cdot$ & + & $\cdot$ & $\cdot$ & + & $\cdot$ & Ad-Vector & $\cdot$ & + & $\cdot$ & $\cdot$ & + & $\cdot$ \\
\hline CIC-3 shRNA & - & $\cdot$ & + & - & - & + & Ad-CIC-3 & $\cdot$ & - & + & $\cdot$ & $\cdot$ & + \\
\hline p47phox & +1 & +5 & & - & $m$ & & p47phox & 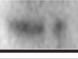 & Wis: & $=$ & wer & sit. & \\
\hline p67phox & 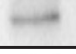 & 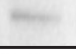 & $m=$ & - & - & $\rightarrow$ & p67phox & $=$ & 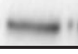 & - & - & -1 & $=$ \\
\hline ATPase & - & 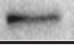 & rents & - & $m$ & $\rightarrow$ & ATPase & 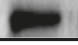 & 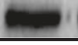 & 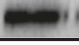 & $=$ & 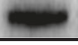 & \\
\hline
\end{tabular}
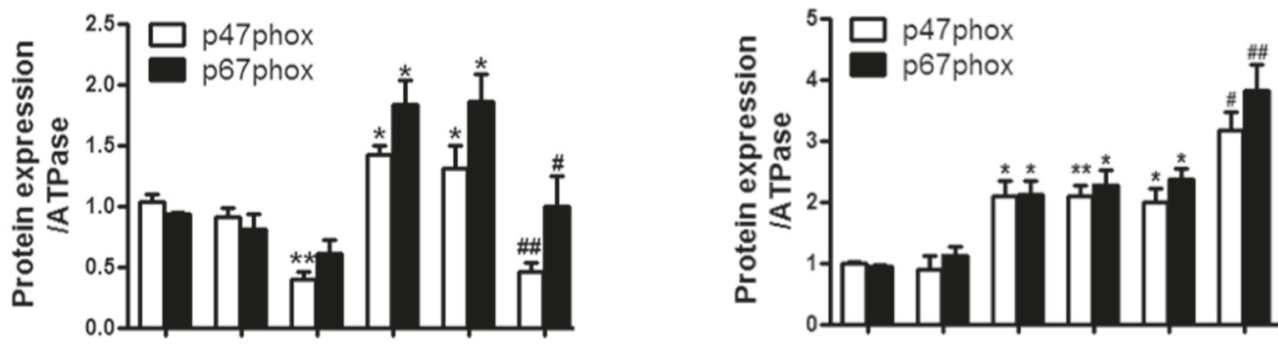

Fig. 4 ClC-3 potentiated Ang II-induced translocation of p47phox and p67phox to the plasma membrane. a and $\mathbf{b}$ Immunoprecipitation showed the interaction of p47phox with p22phox (a) or p67phox (b) in HUVECs treated with or without Ang II (1 $\mu$ mol/L) for $24 \mathrm{~h}$. The cell lysates were immunoprecipitated with anti-p22phox or anti-p67phox antibody and then immunoblotted with anti-p47phox antibody. Knockdown of CIC-3 attenuated, and overexpression of CIC-3 facilitated, Ang II-induced interaction of p47phox with p22phox or p67phox $\left(n=5,{ }^{*} P<0.05\right.$ vs. CON, ${ }^{\#} P<0.05$ vs. Ang II, ${ }^{* *} P<0.01$ vs. CON, ${ }^{\# \#} P<0.01$ vs. Ang II). $\mathbf{c}$ and $\mathbf{d}$ Western blotting measurements showed that knockdown of CIC-3 decreased (c), whereas overexpression of CIC-3 enhanced (d), Ang Il-induced expression of p47phox and p67phox on the cell plasma membrane fraction ( $n=6,{ }^{*} P<0.05$ vs. CON, ${ }^{\#} P<0.05$ vs. Ang II, ${ }^{* *} P<0.01$ vs. CON, ${ }^{\# \#} P<0.01$ vs. Ang II) 
with p22phox. This suggestion was further demonstrated by the expression of p47phox and p67phox in the cell membrane. As shown in Fig. 4c, d, knockdown of $\mathrm{ClC}-3$ decreased the Ang IIinduced expression of p47phox and p67phox in the membrane fraction, whereas the overexpression of $\mathrm{ClC}-3$ produced the opposite effect. These findings indicate that $\mathrm{ClC}-3$ promotes the translocation of p47phox and p67phox from the cytoplasm to the cell membrane and subsequent association with membrane subunits, thus potentiating the assembly and activation of NADPH oxidase in endothelial cells.

\section{ClC-3 increases p47phox phosphorylation}

The general view of NADPH oxidase activation initiates from p47phox phosphorylation, which leads to translocation of the p47phox/p67phox complex to the plasma membrane, where p47phox interacts with p22phox and initiates the formation of the active, membrane-bound enzyme complex [18]. To further understand the mechanism underlying the effects of $\mathrm{ClC}-3$ on NADPH oxidase activation, we examined the phosphorylation of p47phox. Treatment with Ang II increased p47phox phosphorylation in HUVECs, which started 15 min after Ang II administration (Fig. 6a). However, the Ang Il-induced phosphorylation of p47phox was attenuated significantly in ClC-3 shRNA-treated cells (Fig. 5a) and increased in $\mathrm{ClC}$-3-overexpressing cells (Fig. 5b) compared with phosphorylation in the non-transfected groups. Moreover, consistent with the increased NADPH oxidase activity (Fig. 2b), p47phox phosphorylation was also increased by $\mathrm{ClC}-3$ overexpression alone independently of Ang II stimulation (Fig. 5b). These data suggest that facilitating p47phox phosphorylation might account for the effect of $\mathrm{ClC}-3$ on NADPH oxidase activation.

CIC-3 increases p47phox phosphorylation through the p38 MAPK pathway

The activation of mitogen-activated protein kinase (MAPK) signaling, particularly p38 MAPK, has been shown to play an important role in the regulation of p47phox phosphorylation $[19,20]$. To identify the possible signaling pathway underlying the effects of $\mathrm{ClC}-3$ on NADPH oxidase activation, p38 MAPK phosphorylation was further examined. Treatment with Ang II increased the phosphorylation of p38 MAPK in HUVECs in a manner similar to that of p47phox, with an earlier starting time of 5 min after Ang II administration (Fig. 6a), Moreover, consistent with the changes caused by $\mathrm{ClC}-3$ in p47phox

a

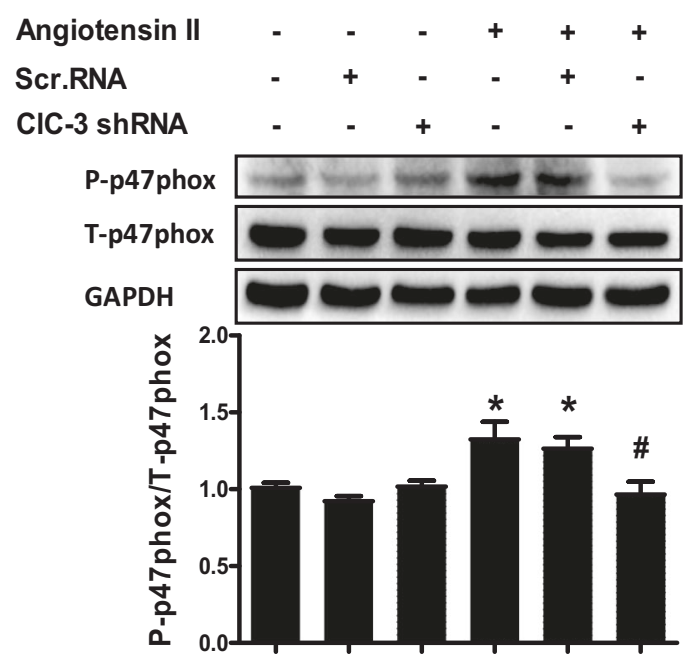

phosphorylation, Ang II-induced phosphorylation of p38 MAPK was attenuated by $\mathrm{ClC}-3$ knockdown and increased by $\mathrm{ClC}-3$ overexpression (Fig. 6b, c).

To further demonstrate the causal link between p38 activation and p47 phosphorylation, we performed additional experiments using the p38 inhibitor SB203580 in CIC-3-overexpressing HUVECs. The results showed that transfection with $\mathrm{Ad}-\mathrm{ClC}-3$ significantly increased p47phox phosphorylation in Ang II-treated HUVECs. However, this effect caused by $\mathrm{ClC}-3$ was abrogated by pretreatment with the p38 inhibitor (Fig. 6d). Accordingly, increased NADPH oxidase activity and ROS production in $\mathrm{ClC}-3-$ overexpressing cells was also abolished by blockage of the p38 signaling pathway (Fig. 6e, f). These results confirmed our proposed mechanistic pathway through which $\mathrm{ClC}-3$ might affect Nox-derived ROS formation by regulating p38 MAPK-dependent p47phox phosphorylation.

\section{DISCUSSION}

In our present study, we explored the correlation between the ClC3 and NADPH oxidase activation induced by Ang II in endothelial cells. The major findings of this study are as follows. (1) $\mathrm{ClC}-3$ is required for NADPH oxidase-derived ROS generation in endothelial cells. CIC-3 silencing inhibits Ang II-induced ROS production, whereas $\mathrm{ClC}-3$ overexpression shows the opposite effect. (2) The pro-oxidative effects of $\mathrm{ClC}-3$ on NADPH oxidase occur by facilitating the translocation of p47phox/p67phox to the plasma membrane as well as the upregulation of Nox2 and p22phox expression. (3) CIC-3 increases NADPH oxidase activity in a p38 MAPK-dependent pathway.

Increased ROS levels play an important role in the development of vascular dysfunction in cardiovascular diseases including hypertension [21]. In recent years, it was demonstrated that the Nox, a membrane-localized protein, is one of the major sources of ROS in the vasculature [14, 22]. There is strong evidence that Ang II, a major member of the renin-angiotensin-aldosterone system, stimulates endothelial ROS formation by upregulating Noxderived ROS production [23]. Consistently, our present study showed that Ang II treatment increased endothelial NADPH oxidase activity and ROS production and induced cell senescence in HUVECs, further demonstrating that Nox is an important culprit for Ang II-induced endothelial injury. The most interesting finding

\section{b}

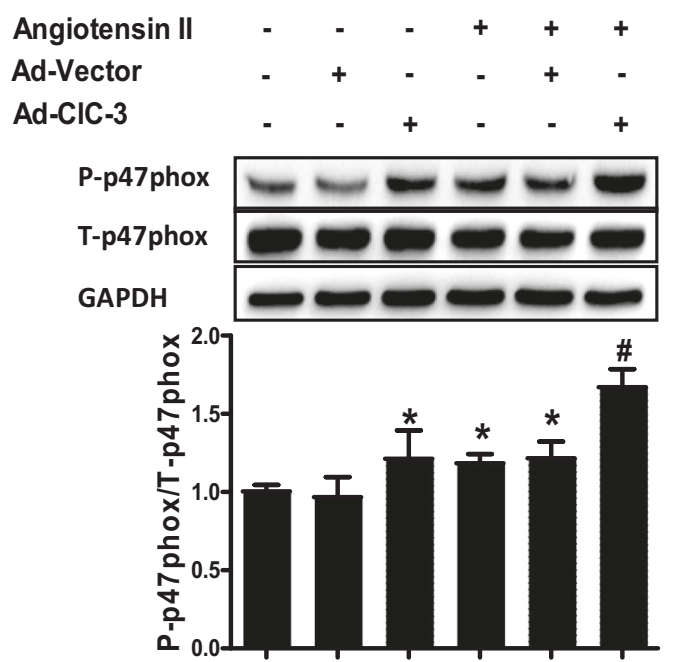

Fig. $5 \mathrm{ClC}-3$ increased p47phox phosphorylation in HUVECs. HUVECs were treated with CIC-3 shRNA or Ad-ClC-3 for $48 \mathrm{~h}$ and then were incubated with or without Ang II (1 $\mu \mathrm{mol} / \mathrm{L})$ for $30 \mathrm{~min}$. Ang II-induced p47phox phosphorylation was decreased by knockdown of ClC-3 (a) and enhanced by overexpression of $\mathrm{ClC}-3$ (b) $\left(n=5,{ }^{*} P<0.05\right.$ vs. CON, ${ }^{\#} P<0.05$ vs. Ang II). Quantification data are presented as the mean \pm SEM in relative units 
1732

of this work, however, is that these changes induced by Ang II are significantly inhibited by $\mathrm{ClC}-3$ knockdown and further potentiated by $\mathrm{ClC}-3$ overexpression, suggesting the requirement of $\mathrm{ClC}$ 3 for NADPH oxidase-derived ROS generation in endothelial cells. Over the past decade, the correlation between $\mathrm{ClC}-3$ and intracellular ROS generation has been suggested in several studies, in which CIC-3 has been shown to be involved in Noxdependent oxidative stress in vascular smooth muscle cells, neutrophils, eosinophils and endothelial progenitor cells [10-13]. The present study, to our knowledge, is the first to provide evidence that Ang II-induced Nox activity and signaling require $\mathrm{CIC}-3$ in endothelial cells.

An important question that remains to be answered is how Nox is regulated by $\mathrm{ClC}-3$. Although the previous studies mentioned the above reported modulation of Nox-derived ROS generation by $\mathrm{ClC}-3$ [10-13], the underlying mechanisms remained to be determined. It has been speculated that $\mathrm{ClC}-3$ might work to compensate for the charge imbalance generated by $\mathrm{O}_{2}{ }^{-}$production from Nox1 and prevent the accumulation of negative charges in the endosomes of vascular smooth muscle cells [11]. However, owing to a lack of evidence, this interpretation remains controversial, as a large body of evidence shows that charge compensation for Nox, including Nox1 and Nox2, results from proton channels, with little or no chloride conductance $[24,25]$. In our present study, we showed that treatment with Ang II remarkably increased the expression of the membrane-bound subunits p22phox as and Nox2, the key catalytic subunit of NADPH oxidase, which is consistent with the
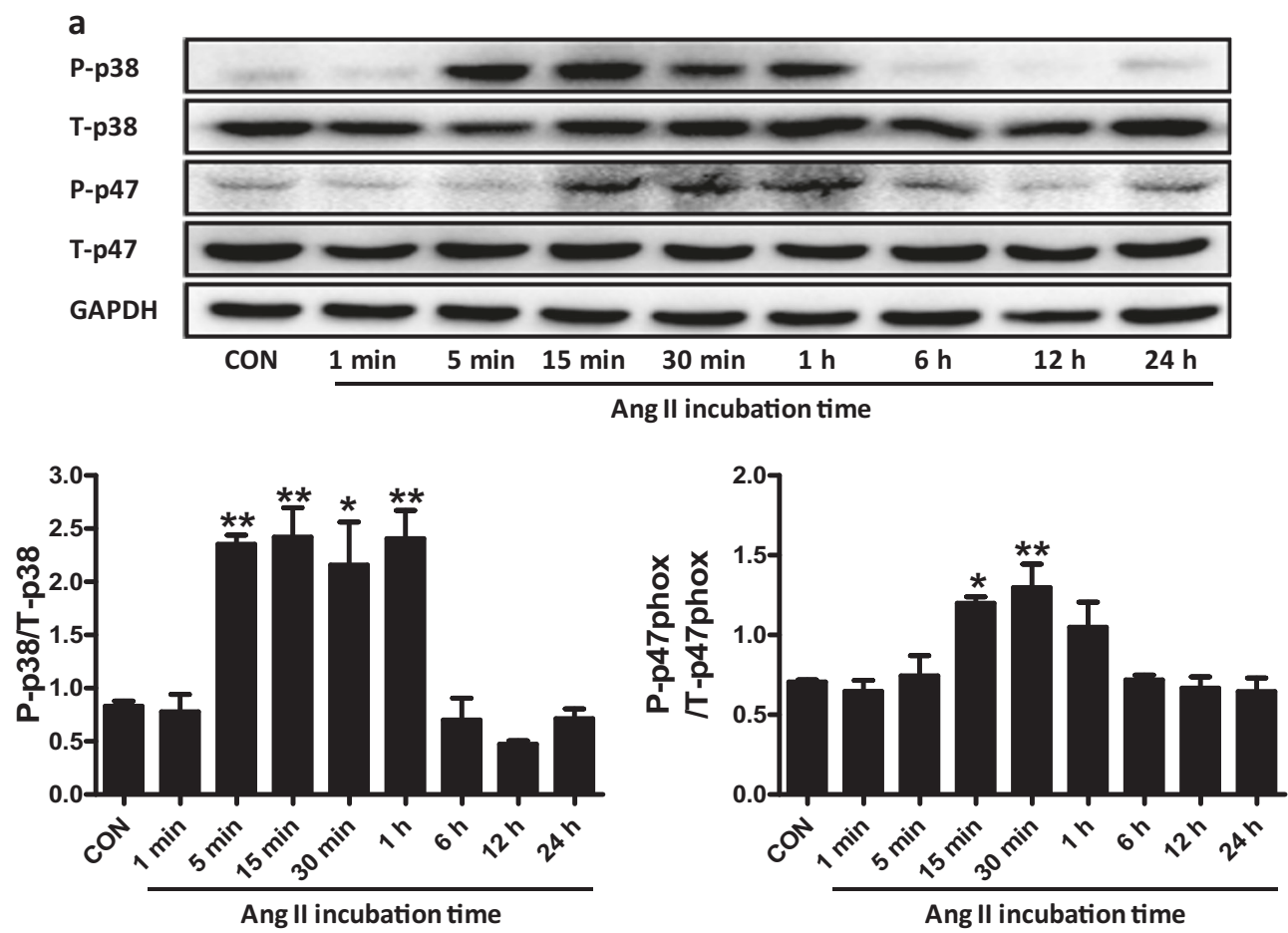

b
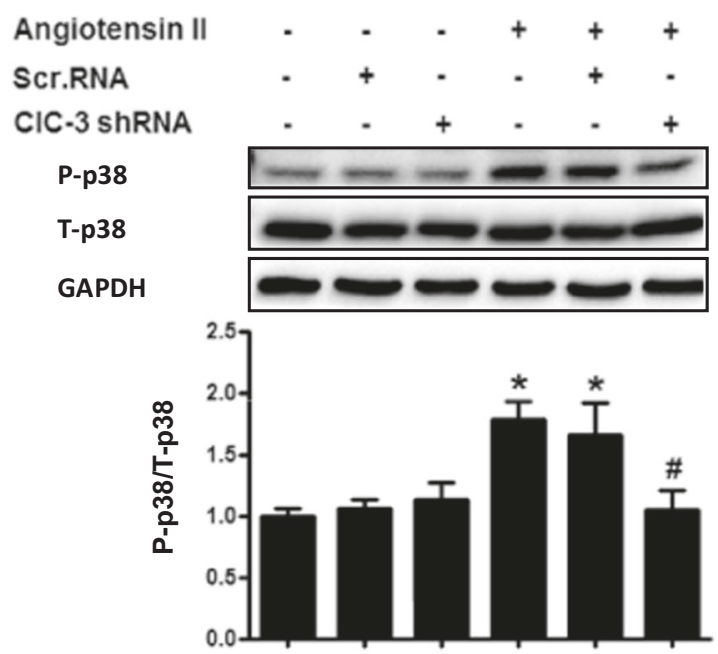

c

Angiotensin II
Ad-Vector
Ad-CIC-3
P-p38
T-p38

GAPDH
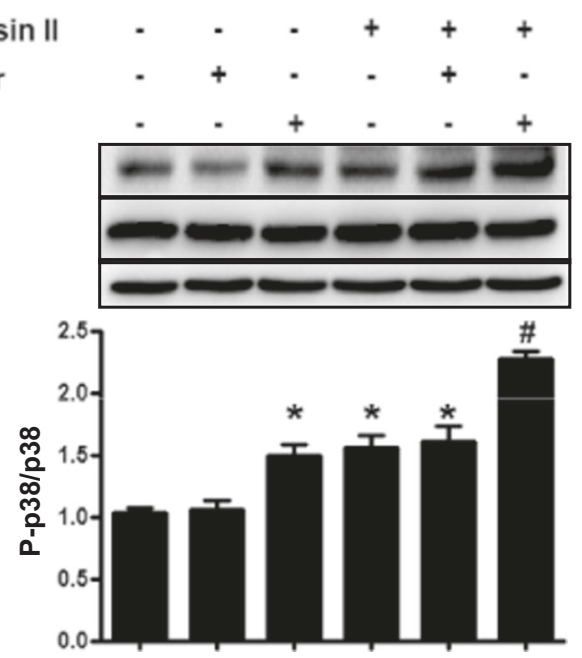


\section{d}

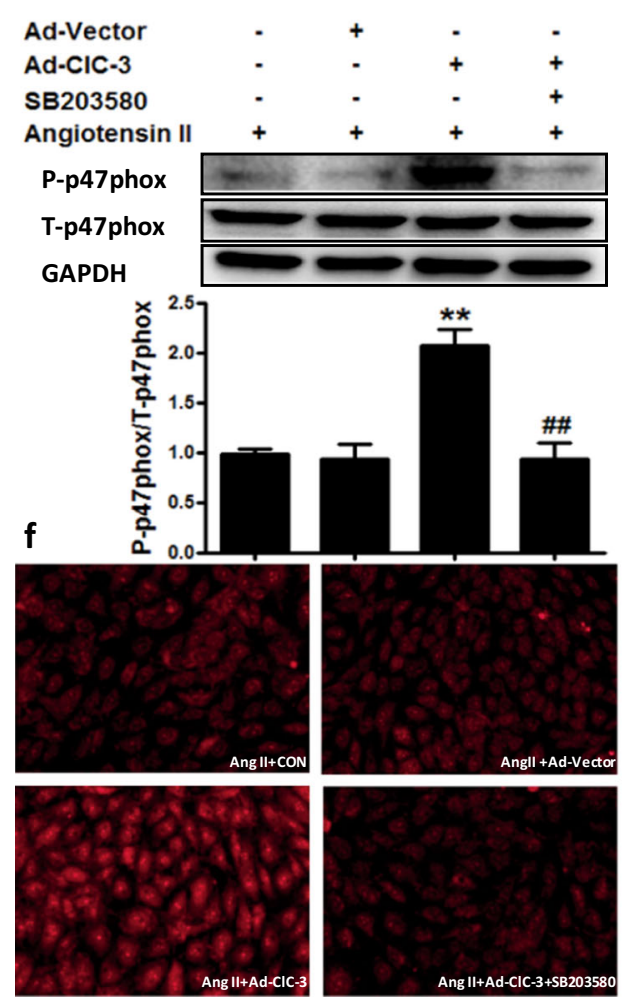

e
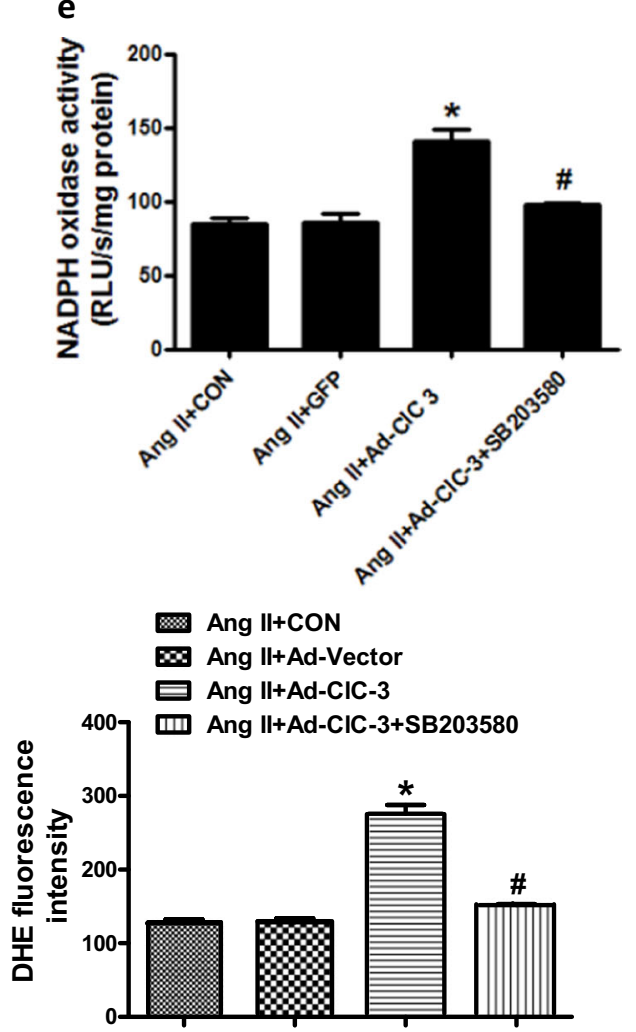

Fig. 6 CIC-3 increased p47phox phosphorylation through p38 MAPK pathway. a The time course of p47phox and p38 MAPK phosphorylation in Ang II-treated HUVECs $\left(n=5,{ }^{*} P<0.05\right.$ vs. CON, ${ }^{*} P<0.01$ vs. CON). b Knockdown of CIC-3 decreased p38 MAPK phosphorylation induced by Ang II $(1 \mu \mathrm{mol} / \mathrm{L})$ treatment for $30 \mathrm{~min}$. c Overexpression of ClC-3 enhanced p38 MAPK phosphorylation $\left(n=5,{ }^{*} P<0.05\right.$ vs. CON, ${ }^{\#} P<0.05$ vs. Ang II). d-f Cells were pretreated with the p38 MAPK inhibitor SB203580 (10 $\mu \mathrm{mol} / \mathrm{L})$ for $6 \mathrm{~h}$ before Ad-CIC-3 transfection. SB203580 abolished the increase in p47phox phosphorylation (d), NADPH oxidase activity (e), and ROS generation (f) induced by CIC-3 overexpression in HUVECs with Ang II treatment, respectively $\left(n=6,{ }^{* *} P<0.01\right.$ vs. Ang II, ${ }^{\#} P<0.01$ vs. Ang II+Ad-CIC-3)

previous reports that Ang II is a well-established stimulus for Nox2 upregulation in arteries [23, 26, 27]. Importantly, this increase in Nox2 was significantly inhibited by knockdown of CIC-3 and further increased by overexpression of CIC-3, indicating that promoting Ang II-induced Nox2 expression might be one of the mechanisms of $\mathrm{ClC}-3$ modulation of Nox activity.

More importantly, it is well known that for Nox2 activation, translocation of the cytosolic subunits p47phox and p67phox to the biological membrane is necessary, where they interact with the membrane-bound subunits to form the active enzyme complex $[17,18]$. In view of this, our results showed that although the total expression levels of p47phox and p67phox were not altered by ClC-3, the Ang II-induced translocation of these two subunits to the plasma membrane, as demonstrated by increased membrane expression and interaction with p22phox, was significantly inhibited by $\mathrm{ClC}-3$ silencing and further potentiated by $\mathrm{ClC}-3$ overexpression. Given the pivotal role of p47phox/p67phox translocation in Nox activation, it is reasonable to suggest that facilitating p47phox and p67phox translocation might play an essential role in the upregulation of Nox activity by $\mathrm{ClC}-3$. This implication is of great importance because the cytosolic subunit translocation appears to be a general process for several Nox isoform activation. Regarding the present study, it is likely that at least for Nox2-containing NADPH oxidase in endothelial cells, by upregulation of the cytosolic subunits translocation, together with increased membrane-bound subunits expression, $\mathrm{ClC}-3$ potentiates the assembly of NADPH oxidase subunits in the membrane and thus promotes the activation of the enzyme complex.
A number of studies have revealed that the translocation of cytosolic subunits is initiated from p47phox phosphorylation $[18,28]$. The activation of p38 MAPK has been shown to contribute to the phosphorylation of p47phox $[19,20]$. In our previous study, $\mathrm{CIC}-3$ was found to be an essential regulator of $\mathrm{p} 38$ phosphorylation via the reduction of intracellular chloride concentration $\left(\left[\mathrm{Cl}^{-}\right]_{\mathrm{i}}\right)$ through $\mathrm{ClC}-3$-dependent $\mathrm{Cl}^{-}$efflux in foam cells [8]. Based on these implications, another interesting finding of our study is that both p47phox and p38 MAPK phosphorylation induced by Ang II were significantly inhibited by CIC-3 knockdown. Moreover, pretreatment with the p38 MAPK inhibitor SB203580 remarkably abrogated the increase in p47phox phosphorylation as well as the NADPH oxidase activity and ROS production induced by $\mathrm{ClC}-3$ overexpression. These results indicated that there is a close causal link between $\mathrm{ClC}-3$ and p38 MAPK/p47phox phosphorylation. CIC-3 might facilitate active NADPH oxidase complex formation via the upregulation of p38 MAPK-dependent p47phox phosphorylation.

It is also noteworthy that p47phox phosphorylation and translocation as well as Nox activity and ROS production were all increased in ClC-3-overexpressing cells without Ang II treatment, indicating that $\mathrm{ClC}-3$ might be an independent risk factor for oxidative stress. A high level of $\mathrm{ClC}-3$ alone is sufficient to cause Nox-derived ROS production. It has been shown that CIC3 expression is upregulated in various cardiovascular diseases featured by increased oxidative stress, including hypertension [29], atherosclerosis [8], and diabetes [30]. Consistently, our present study also showed an increase in ClC-3 expression in Ang II-treated endothelial cells. Therefore, the inhibition of $\mathrm{ClC}-3$ 
might be a rational therapeutic strategy to treat ROS-associated diseases.

In summary, our findings reveal that $\mathrm{ClC}-3$ is required for Nox activation by Ang II in endothelial cells. Promoting the formation of the active enzyme complex of Nox2 might represent an important mechanism for the dependence of Nox on $\mathrm{ClC}-3$. The present study might provide new mechanistic insights for understanding how $\mathrm{ClC}-3$ regulates Nox activity in the vasculature and might therefore open the door to new therapy for cardiovascular disease.

\section{ACKNOWLEDGEMENTS}

This work was supported by the National Natural Science Foundation of China (No. 81373401 and 81573422); Natural Science Foundation of Guangdong Province, China (No. S2013010016611); and the Fundamental Research Funds for the Central Universities (No. 2015ykzd01).

\section{AUTHOR CONTRIBUTIONS}

Y-hD and G-zL designed the study and drafted the manuscript; G-zL and L-mC performed the experiments; $Y$-yG assisted with the data analyses and discussion; other colleagues assisted with the experiments and data collection.

\section{ADDITIONAL INFORMATION}

Competing interests: The authors declare no competing interests.

\section{REFERENCES}

1. Lambeth JD. Nox/Duox family of nicotinamide adenine dinucleotide (phosphate) oxidases. Curr Opin Hematol. 2002;9:11-7.

2. Lassegue $B$, Clempus RE, Vascular. NAD(P)Hoxidases: specific features, expression, and regulation. Am J Physiol. 2003;285:R277-97.

3. Duan D, Winter C, Cowley S, Hume JR, Horowitz B. Molecular identification of a volume-regulated chloride channel. Nature. 1997;390:417-21.

4. Jentsch TJ. Chloride and the endosomal-lysosomal pathway: emerging roles of CLC chloride transporters. J Physiol (Lond). 2007;578(pt 3):633-40.

5. Duran C, Thompson CH, Xiao Q, Hartzell HC. Chloride channels: often enigmatic, rarely predictable. Annu Rev Physiol. 2010;72:95-121.

6. Guan YY, Wang GL, Zhou JG. The $\mathrm{ClC}-3 \mathrm{Cl}$ - channel in cell volume regulation, proliferation and apoptosis in vascular smooth muscle cells. Trends Pharmacol Sci. 2006;27:290-6.

7. Chu X, Filali M, Stanic B, Takapoo M, Sheehan A, Bhalla R, et al. A critical role for chloride channel-3 (CIC-3) in smooth muscle cell activation and neointima formation. Arterioscler Thromb Vasc Biol. 2011;31:345-51.

8. Tao J, Liu CZ, Yang J, Xie ZZ, Ma MM, Li XY, et al. ClC-3 deficiency prevents atherosclerotic lesion development in $\mathrm{ApoE}^{-1-}$ mice. J Mol Cell Cardiol. 2015;87:237-47.

9. Zheng LY, Li L, Ma MM, Liu Y, Wang GL, Tang YB, et al. Deficiency of volumeregulated $\mathrm{CIC}-3$ chloride channel attenuates cerebrovascular remodelling in DOCA-salt hypertension. Cardiovasc Res. 2013;100:134-42.

10. Moreland J, Davis A, Bailey G, Nauseef W, Lamb F. Anion channels, including CIC3 , are required for normal neutrophil oxidative function, phagocytosis, and transendothelial migration. J Biol Chem. 2006;281:12277-88.
11. Miller F, Filali M, Huss G, Stanic B, Chamseddine A, Barna T, et al. Cytokine activation of nuclear factor $B$ in vascular smooth muscle cells requires signaling endosomes containing Nox1 and CIC-3. Circ Res. 2007;101: 663-71.

12. Gaurav R, Bewtra AK, Agrawal DK. Chloride channel 3 channels in the activation and migration of human blood eosinophils in allergic asthma. Am J Respir Cell Mol Biol. 2015;53:235-45.

13. Liu J, Zhang FF, Li L, Yang J, Liu J, Guan YY, et al. CIC-3 deficiency prevents apoptosis induced by angiotensin II in endothelial progenitor cells via inhibition of NADPH oxidase. Apoptosis. 2013;18:1262-73.

14. Bedard K, Krause KH. The NOX family of ROS-generating NADPH oxidases: physiology and pathophysiology. Physiol Rev. 2007;87:245-313.

15. Yang $H$, Huang LY, Zeng DY, Huang EW, Liang SJ, Tang YB, et al. Decrease of intracellular chloride concentration promotes endothelial cell inflammation by activating nuclear factor-KB pathway. Hypertension. 2012;60:1287-93.

16. Dikalov $\mathrm{SI}$, Nazarewicz RR, Bikineyeva A, Hilenski L, Lassègue $B$, Griendling KK, et al. Nox2-induced production of mitochondrial superoxide in angiotensin IImediated endothelial oxidative stress and hypertension. Antioxid Redox Signal. 2014;20:281-94.

17. Lambeth JD. NOX enzymes and the biology of reactive oxygen. Nat Rev Immunol. 2004:4:181-9.

18. Groemping Y, Lapouge K, Smerdon SJ, Rittinger K. Molecular basis of phosphorylation-induced activation of the NADPH oxidase. Cell. 2003;113: 343-55.

19. Parinandi NL, Kleinberg MA, Usatyuk PV, Cummings RJ, Pennathur A, Cardounel $A J$, et al. Hyperoxia-induced $\mathrm{NAD}(\mathrm{P}) \mathrm{H}$ oxidase activation and regulation by MAP kinases in human lung endothelial cells. Am J Physiol Lung Cell Mol Physiol. 2003;284:L26-38.

20. Lal AS, Clifton AD, Rouse J, Segal AW, Cohen P. Activation of the neutrophil NADPH oxidase is inhibited by SB 203580, a specific inhibitor of SAPK2/p38. Biochem Biophys Res Commun. 1999;259:465-70.

21. Lassegue B, Griendling KK. Reactive oxygen species in hypertension: an update. Am J Hypertens. 2004;17:852-60.

22. Li JM, Shah AM. Endothelial cell superoxide generation: regulation and relevance for cardiovascular pathophysiology. Am J Physiol Regul Integr Comp Physiol. 2004;287:R1014-30.

23. Schulz E, Gori T, Münzel T. Oxidative stress and endothelial dysfunction in hypertension. Hypertens Res. 2011;34:665-73.

24. Murphy R, DeCoursey T. Charge compensation during the phagocyte respiratory burst. Biochim Biophys Acta. 2006;1757:996-1011.

25. Lassegue B. How does the chloride/proton antiporter $\mathrm{CIC}-3$ control NADPH oxidase? Circ Res. 2007;101:648-50.

26. Schuhmacher $S$, Foretz $M$, Knorr $M$, Jansen $T$, Hortmann $M$, Wenzel $P$, et al. a1AMP-activated protein kinase preserves endothelial function during chronic angiotensin II treatment by limiting Nox2 upregulation. Arterioscler Thromb Vasc Biol. 2011;31:560-6.

27. Mollnau $H$, Wendt $M$, Szocs $K$, Lassegue $B$, Schulz $E$, Oelze $M$, et al. Effects of angiotensin II infusion on the expression and function of $N A D(P) H$ oxidase and components of nitric oxide/cGMP signaling. Circ Res. 2002;90: E58-65.

28. Fisher AB. Redox signaling across cell membranes. Antioxid Redox Signal. 2009;11:1349-56.

29. Liu YJ, Wang XG, Tang YB, Chen JH, Lv XF, Zhou JG, et al. Simvastatin ameliorates rat cerebrovascular remodeling during hypertension via inhibition of volumeregulated chloride channel. Hypertension. 2010;56:445-52.

30. Huang YY, Huang XQ, Zhao LY, Sun FY, Chen WL, Du JY, et al. CIC-3 deficiency protects preadipocytes against apoptosis induced by palmitate in vitro and in type 2 diabetes mice. Apoptosis. 2014;19:1559-70. 